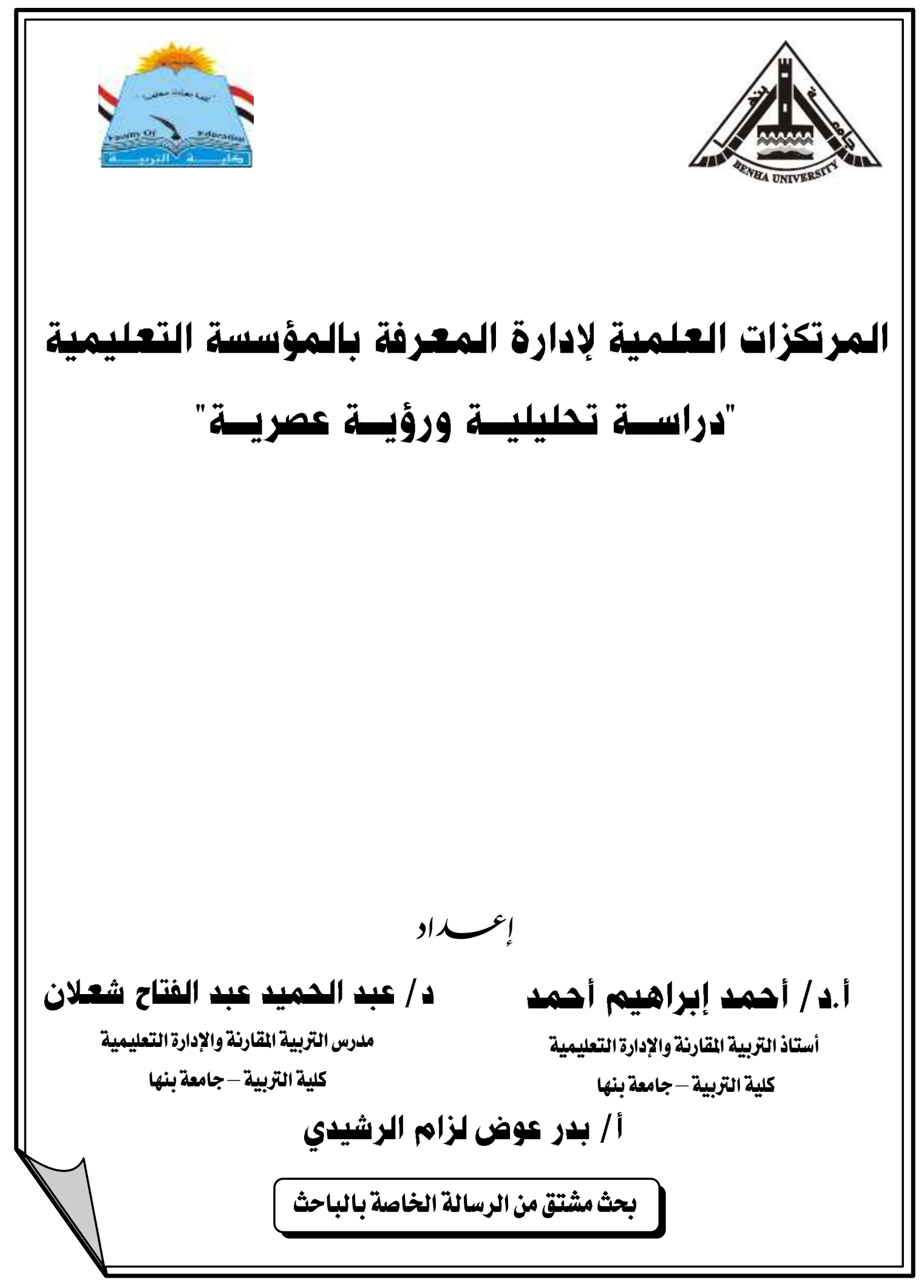




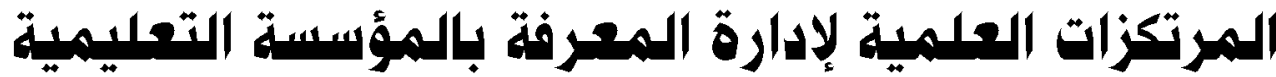

"دراسة تحليلية ورؤية عصريةت"

\author{
! إ
}

د/ عبد الدمبـ عبد الفتاح شعسلان

مدرس التربية المقارنة والإدارة التمليمية

كلية التربية - جامعة بنها

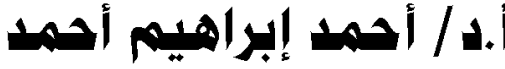

أستاذ التربية المقارنة والإدارة التعليمية

كلية التربية - جامعة بنها

أ/ بلدر هوض لزام الرشيدي

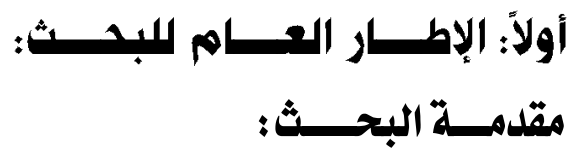

يعتبر مصطلح "إدارة المعرفة" مصطلحا حديثا، لذا فقد حرصت عليها الحضـارات عبر عصور التاريخ المختلفة، وتطبيق إدارة المعرفة في المؤسسة المعاصرة فتح لها أفاقا واسعة نحو تعزيز مكانتها وإمكاناتها وقدراتها التنافسية، لأنها أداة فاعلة تساعدها على الدخول في عصر المعرفة والمعلوماتية. والتحول الكبير في أساليب عمل المؤسسة بعدما أصبحت المعرفة جزءا مهما في رأسمالها، ساهم في ظهور أسلوب إداري جديد يسعي إلي مواكبة العصر الجديد بغية التمتع ببعض المزايا التى تجعلها تتفوق على المنافسين في السوق(').

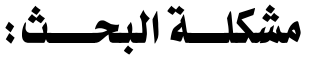

يشهد القرن الحالي تطورات هائلة في مختلف المجالات ومن بين أهمها مجالات الأعمال والاقتصاد، حيث أصبحت تمثل اهتماما كبيرا لمؤسسات الأعمال، ومن أبرز هذه التطورات ظاهرة العولمة والتحول نحو اقتصاد المعرفة، هذا الاقتصـاد الذي لم تعد فيه الأرض واليد العاملة ورأس المال الموارد الأساسية، بل ظهر عنصر رابع أصبح يعتبر المورد الأهم للاقتصساد والمتمثل في المعرفة باعتبارها نوعاً جديداً من عناصر الإنتاج ونوعاً من رأس المال القائم على المعرفة والخبرة، وهو رأس المال الفكري الذي يتجدد ويتطور باستمرار (؟). 
وعلى هذا يمكن صياغة مشكلة في السؤال الرئيس التالي:

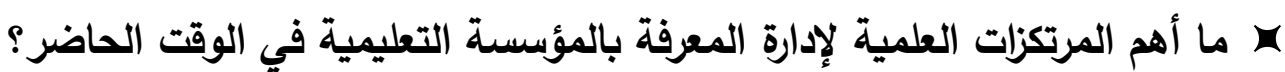
ويتفرع عنه التساؤلات الفرعية التالية :

" ما أهم المضامين العلمية للمعرفة بالمؤسسة التعليمية؟ ما أهم الأسس النظرية لإدارة المعرفة للمؤسسة التعليمية؟

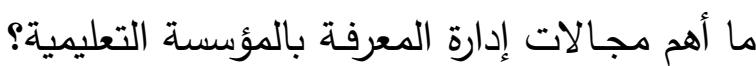
ما أهم نماذج إدارة المعرفة بالمؤسسة التعليمية؟ • كيف تستثيد المؤسسة التعليمية من نماذج إدارة المعرفة؟

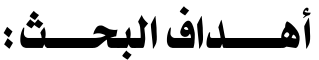

استهدف البحث الحالي تحديد المرتكزات العلمية لإدارة المعرفة بالمؤسسة التعليمية في

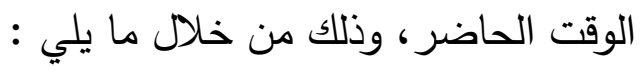
ا التعرف على أهم المضامين العلمية للمعرفة بالمؤسسة التعليمية. الكثف عن أهم الأسس النظرية لإدارة المعرفة للمؤسسة التعليمية. • الوقوف على أهم مجالات إدارة المعرفة بالمؤسسة التعليمية. " تحديد أهم نماذج إدارة المعرفة بالمؤسسة التعليمية. تحديد كيفية استفادة المؤسسة التعليمية من نماذج إدارة المعرفة.

\section{منهــــج البحــــث:}

استخذم البحث الحالي المنهج الوصفي حتى يحقق أهدافه العلمية ويجيب عن تساؤلاته البحثية.

\section{ثانياً: المضامين العلمية للمعرفة بالمؤسسة التعليمية :}

قبل التطرق إلى مفهوم المعرفة يجب المرور بمراحل المعرفة وهي بيانات معلومات ثم معرفة(ץ). • البيانات وهي المادة الخام الأولية التي تستخلص منها المعلومات وقد تظهر في شكل أرقام أو حروف أو إثارات أو صورة دون سابق أو تتظيم لها وتصبح البيانات معلومات عندما يتم تصنيفها وتتقيحها وتحليلها ووضعها في إطار واضح ومفهوم (ء). • المعلومـات وهي مجموعة من البيانات المعالجة والمؤطرة والمؤسسة والمترابطة والمعدة

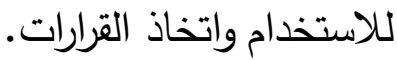


• المعرفة وهي أحد العناصر الأساسية ضمن سلسلة متكاملة، تبدأ بالإشارات، ثم إلى معلومات، ثم إلى معرفة، ثم إلى حكمة وهذه الأخيرة هي أساس الابتكار ، وتعرف المعرفة أيضا : "معلومات مؤسسة قابلة للاستخدام في حل مشكلة معينة أو هي معلومات مفهومة محللة ومطبقة"(*)، وتعرف أيضا بأنها اسم مشتق من الفعل(عرف) وتشير إلى القدرة على التميز أو التلاؤم وهي إذن كل ما هو معرف أو ما هو مفهوم (־)، وتعرف كذلك :

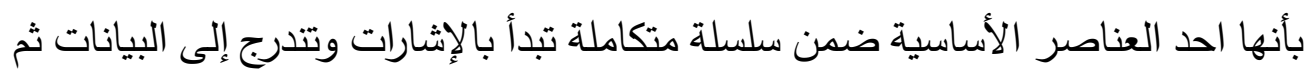
إلى معلومات ثم إلى معرفة ثم إلى حكمة وهذه الأخيرة هي أساس الابتكار (v). ومن خلال هذه التعاريف نصل إلى أن المعرفة هي نتائج معالجة البيانات التي تخرج بمعلومات إذ تصبح معرفة بعد استيعابها وفهمه وتكرار تطبيقها في الممارسات ويؤدي ذلك إلى خبرة والتي تؤدي بدورها إلى حكمة، والتي تجسد الذكاء وفهم ما هو صحيح وخطأ، وما هو حقيقي وزائف، وفهم القيمة الدائمة، كما تشمل الحكمة القدرة على قبول التوجهات الجديدة التي يمكن أن

$$
\text { يكون مرغوب فيها ويمكن إدراكها }
$$

\section{وللمعرفة مجموعة من الخصائص يمكن أن نوجزها فيما يلي(^)؛}

المعرفة يمكن أن تولا : بعض المؤسسات لديها خصوبة ذهنية مدا يجعلها قادرة على

توليد المعرفة وهذا ما يمثله الأفراد المبتكرون الذين يبتكرون ويولدون المعرفة. المعرفة يمكن أن تموت : كما تولد المعرفة فإنها تموت بإحلال المعارف الجديدة محل القديمة لتتقاعد عن الاستعمال. " المعرفة يمكن أن تخزن : المعرفة يمكن أن تخزن بشتى الوسائل فقد كانت في العشرين سنة الماضية على الورق، والأشرطة وعلى وسائل التخزين الالكترونية. المعرفة يمكن أن تمتلك : أن المؤسسات تمارس دورا كبيرا في تحويل المعرفة التي تمتلكها إلى براءات اختراع أو أسرار تجارية تتمتع بالحماية القانونية شأن الملكية المادية.

\section{وهناك من يصنف خصائص أخرى للمعرفة وهي (9):} تجدد المعرفة : المعرفة متطورة بشكل دائم بسبب تطور العقول وتحسينها مـع

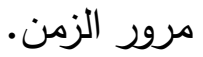
إنتاج المعرفة : بواسطة الأفراد المبتكرون للأفكار الجديدة والذين يتم الاعتماد عليهم في عملية الإبداع. - ملع. 
• تصنيف المعرفة : تصنف المعرفة بأشكال عديدة وأنماط مختلفة حسب طبيعتها أو استخدامها أو هدفها. وتبرز أهمية المعرفة من خلال إضافة قيمة للمؤسسة، وكذا في الدور الذي تؤيده في تحويل المؤسسة إلى اقتصاد المعرفة، ويمكن أن نحدد هذه الأهمية في النقاط التالية(· '): المعرفة أصبحت أساس لخلق ميزة تنافسية واستخدامها. توجه المعرفة الإدارية ددراء المؤسسات إلى كيفية أداء مهماتهم بكفاءة. المعرفة هي الأساس وراء كيفية تقدم المؤسسة وتطورها ونضجها.

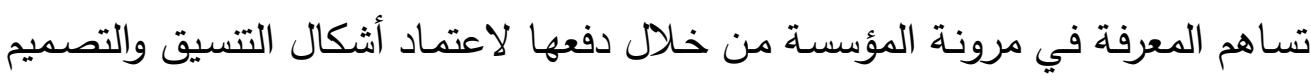
والهيكلة تكون أكثر مرونة.

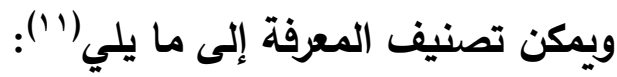

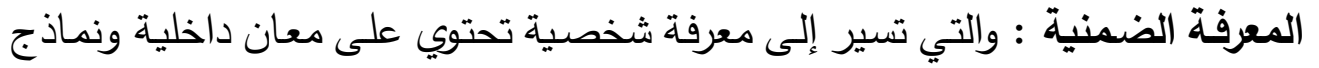
ذهنية وخبرات وتبصر وبديهة وهي نوعان احدهما تقني يعود إلى العمق والأخر إدراكي يحتوي على أفعال وسلوكيات يومية. المعرفة المعلنة : وهي المعرفة التي يعبر عنها من خلال الحقائق والتعبيرات والرسومات والتصورات، ويمكن توثيقها في الورق أو في الثكل الالكتروني. المعرفة التكنولوجية : وهي جزء من المعرفة الضمنية وتعبر عن البراعة والخبرة والمهارة

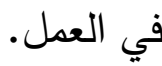
المعرفة الضحلة : وتعني الفهم القليل لمؤشرات مساحات المشكلة. المعرفة العميقة : وهي التي تتطلب التحليل العميق للمواقف المختلفة. المعرفة السلبية : وهي التي تتم بناء على ربط المفاهيم معاً باستخدام طرق الاستتناج والاستقراء. هالمعرفة الموجهة : وهي المعرفة التي تبني على أساس عدد سنوات الخبرة في ومن أبرز تقسيمات المعرفة من ينظر إليها على أنها معرفة ضمنية Taci knowledge ومعرفة التهات صريحةExplicit knowledge على اعتبار أن (r'):

\section{ا- المعرفة الضمنية Taci knowledge}

وهي المعرفة الموجودة في عقول الأفراد غير رسمية والتي يعبر عنها بالطريقة الحدسية والنوعية وغير القابلة للنقل والتعليم، وتوجد في عمل الأفراد والفرق في المؤسسات وهذه المعرفة الدردي 
تعطي للمؤسسة، خصوصيتها وشخصيتها وقدرتها على إنشاء وتوليد معارف جديدة، ووصفت بأنها الخيرات والتجارب لأعضاء المؤسسة التي لم توثق رسميا.

\section{ب : Exiplici knowledge المعرفة الصريحة}

وهي معرفة رسمية نظامية معبر عنها كمياً وقابلة للنقل والتعلم، ويمكن تسربها خارج المؤسسة ونجدها في أشكال فكريـة كما نجدها مجسدة في منتجات المؤسسة وخدماتها ومعايير

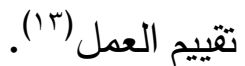

ويوجد نموذج يسمح بالانتقال من المعرفة الصريحة إلى الضمنية أو العكس وتسمى كل حالة من هذه الحالات كما يلي (\&) (): • الاثثتركية Socialization والتي تتضمن التحويل من ضمنية إلى توضيحية وهي من بين الأشكال الأسفل لتبادل المعرفة لأنها تتنقل بصفة عفوية عند كل موضع في العمل، لكن المعرفة في هذه الحالة تبقى في عقول المشاركين فيها ومن الصعب توثيقها. • التوضيحية Externalzation : من الضمنية إلى الصريحة بحيث تأخذ شكل مفاهيم ونماذج تسهل من نقلها والاشتراك فيها. التجميعية combimation : وهي التحول من معرفة صريحة إلى معرفة ضمنية كما في

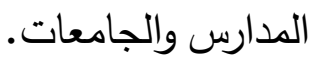
• الاخلية Internalization : والتي تئكد على التحول من معرفة صريحة إلى معرفة ضمنية من خلال تكرار أداء المهمة وتصبح المعرفة الصريحة مستوعبة مثل المعرفة

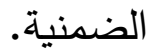
والحصول على المعرفة يقصد به شكل تعلم الإنسان للمعارف وادراكها وهذا يجري بأثكال مختلفة وهي الملاحظة والعقل والمنطق والخبرة والعلمية، ويمكن تقسيم مصادر المعرفة إلى مصادر داخلية ومصادر خارجية، ويمكن عرضها على النحو التالي (10): 1-المصادر الهافلبة لامعرفة :

وهي مصادر تتبع من المؤسسة وتزودها بالمعرفة ويمكن حصرها كما يلي: أ) إستراتيجية المعرفة Knoweledge Stratégiste : هم الذين يعيدون تقييم أو بناء إستراتيجية المؤسسة وهم مسئولون عن الاهتمام بمعرفة الأغراض وهم خبراء الإستراتيجية والمنافسة القائمة على المعرفة في السوق وينفذون بشكل فاعل وعميق الأدوار الجديدة للمعرفة 
داخل المؤسسة يوصفها رأس مال فكري داخل المؤسسة من جهة وخارج المؤسسة، والمعرفة

$$
\text { توليها وتقاسمها هي السلاح الاستراتيجي التنافسي الجديد من جهة أخرى. }
$$

ب) مهنيو المعرفة Knoweledge Professional : وهؤلاء هم الذين يتعلمون مـع المعرفة كموضوع وهم من يستوعبون المضمون المعرفي وهم الخبراء في أساليب الحصول عليها واستيعابها بالمنهجية الفعالة لتوليدها أو جعلها قادرة على العمل في مجالات التهن الاستعمال

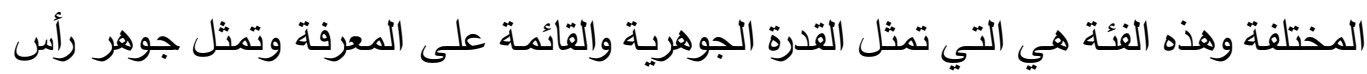
المال الفكري للمنظر وقيمتها المتجدة في توليد وانشاء المعرفة. ج) عمال المعرفة Knoweledge Workers : هؤلاء يعملون في مجال المعرفة من اجل توفير عوامل الدعم والإسناد المهني للمعرفة فهم يقدمون بجمع المعلومات وتصنيفها وجدولتها

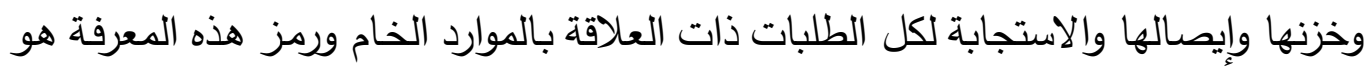

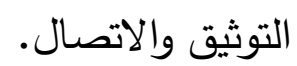

\section{ثالثاً: الأسس النظرية لإدارة المعرفة للمؤسسة التعليمية :}

لقد تتاول الباحثون مفهوم إدارة المعرفة من زوايا عدة، فمنهم من تتاولها من منظور تتني

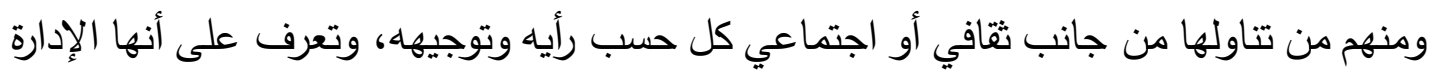
النظامية الواضحة للمعرفة والعمليات المرتبطة بها واستغلالها، وجمعهم وتتظيمها، ونشرها

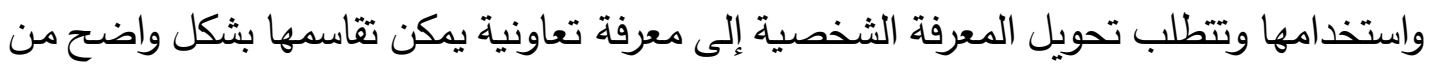

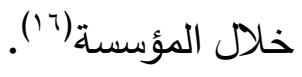
وتعرف أيضاً على أنها تجسيد عملية تتظيمية التي تبحث في عملية مزج قابلية تقنيات

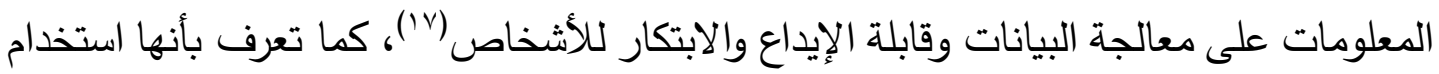

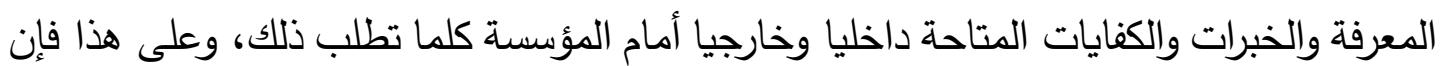

ولإدارة المعرفة أهمية كبيرة يمكن إجمالها في النقاط التالية(19) : • ربط المعل المعارف والمعلومات والخيرات لتطوير المؤسسة. تضاعف حجم المعلومات في كل مجال. " إعادة تثكيل الكفاءات الحيوية. • خلق قواعد حركية لفائدة حقيقية من المعارف في المؤسسة. استخدام مراكز عمل لخلق إدارة المعرفة والحفاظ عليها. 
• تحفيز المؤسسات على تجديد ذاتها ومواجهة التغيرات البيئية الغير مستقرة.

إتاحة الفرصة للحصول على الميزة التتافسية الدائمة للمؤسسة (·r).

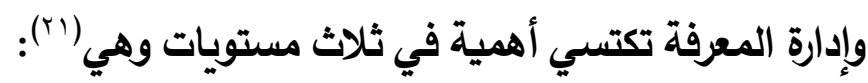

ا - على مستوى الأفراد : تساعد الأفراد إثناء أداء الأعمال بتوفير الوقت من خلال تحسين عملية

اتخاذ القرارات وحل المشكلات وتعزيز مفهمو الروابط المجتمعة داخل المؤسسة، وزيادة فرص المساهمة الفردية في تحقيق الأهداف.

ץ- على مستوى جماعات الممارسـة : تتمية المهارات الوظيفية، تعزيز فعالية الشبكات والعمل

التعاوني والمشاركة بالمعرفة في تطوير لغة المشاركة داخل المؤسسة.

ب- على مستوى تنظيمي : تساهم إدارة المعرفة في قيادة الإستراتيجية وتحقيق أهدافها ونشر أفضل الممارسات داخل المؤسسة وبالتالي تحسين دمج المعرفة، وكذلك زيادة فرص الابتكار

وبناء ذاكرة تتظيمية.

وتهدف إدارة المعرفة إلى تحقيق ما يلي (rr):

تحديد مصادرها وخزنها وإعادة استعمالها.

جذب راس المال فكري اكبر لوضع الحلول للمشكلات التي تواجه المؤسسة.

تحديد المعرفة الجوهرية وكيفية الحصول عليها وحمايتها.

بناءة إمكانية التعلم وإثاعة ثقافة المعرفة والتحفيز لتطويرها والتتافس من خلال الذكاء البشري.

تحويل المؤسسات من الاقتصاد التقليدي إلى اقتصـاد العالمي الجديد (اقتصـاد المعرفة)

الواسعة والتجارة الاككترونية.

جمع الأفكار الذكية في الميدان، ونشر أفضل الممارسة في الداخل.

تحقيق الإبداع والوعي والتصميم الهادف والتكيف مع الاضطراب والتعقيد البيئي والتنظيم

الذاتي والذكاء والتعلم.

التركيز على تتمية الجوانب الاجتماعية والثقافية والتتظيمية لإدارة المعرفة.

وضع بيانات وأدلة خاصة بالإعمال ذات العلاقة بمجتمع المعرفة.

التعريف والتوعية بشكل شمولي لمعنى إدارة وتطوير المعرفة ونشره بين رجال

تطوير أسس ومعايير تأهيل لإدارة المعرفة تساعد في تطوير الجوانب المهنية والتعليمية

للمهنيين المختصين في إدارة المعرفة(rم). 


\section{رابعاً : مجـالات إدارة المعرفهة بالمؤسسة التعليمية :}

تتطلب إدارة المعرفة وجود تتسيق فاعل بين مختلف الموجودات والنشاطات داخل

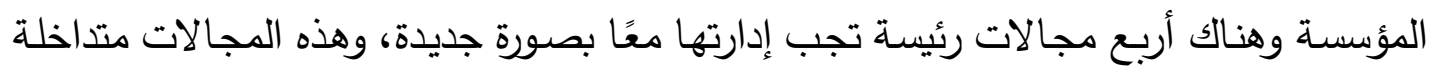

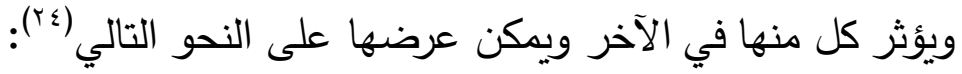

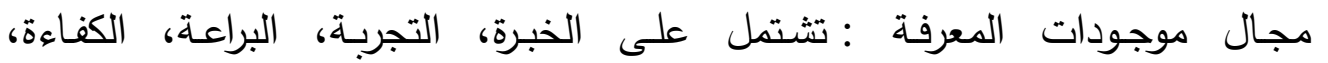

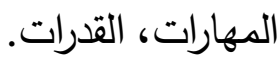
مجال نشاطات المعرفة : يشتمل على عمليات التوليد، البناء، النقل، المراقبة،

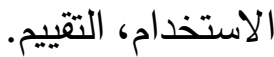

هجال القدرات والميول : يشتمل على قدرات الأفراد وميولهم وقدرات المؤسسة لبناء المعرفة واستخدامها من أجل تحقيق الحد الأعلى من مصالح المؤسسة. مجال المؤسسة أنه يشتمل على أهداف المؤسسة وتوجها وإِتراتيجيتها وممارساتها وثقافتها.

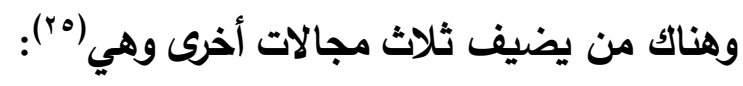
1- مجال الأعمال : الذي يركز على لماذا وأين وإلى أي حد يجب لئ على المؤسسات الاستثمار

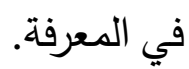
r- مجال الإدارة : الذي يركز على تحديد وتنظيم وتوجيه ومراقبة الأنشطة ذات العلاقة

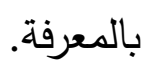
r- مجال العمليات الفعلية :الذي يهتم بتطبيق الخبرات لأداء عمل مبنى على أساس المعرفة.

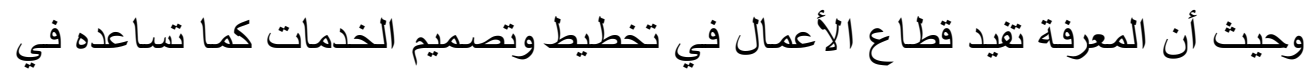

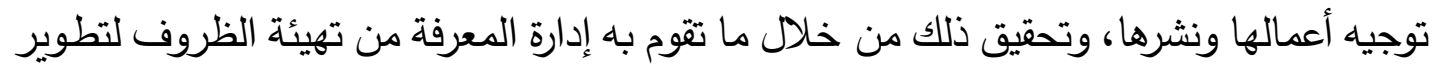
المعرفة واكتسابها ونقلها ومتابعة إدارة عملياتها إلى جانب وضـع السياسات الملائمة لإدارة

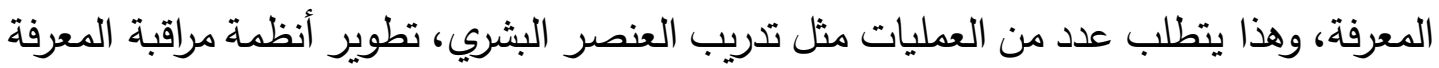
وتكوين شبكات خبرة معرفية.

ودخول المؤسسة التعليمية عصر إدارة المعرفة يتحتم عليها ما يلي :

$$
\begin{aligned}
& \text { 1- استرجاع المعلومات. } \\
& \text { ץ-نظم إدارة المعلومات والمعارف. } \\
& \text { r- مبادئ إدارة المعرفة. } \\
& \text { ع - تتظيم المعلومات. }
\end{aligned}
$$




$$
\begin{aligned}
& \text { 0- تصميم قواعد البيانات. } \\
& \text { 7- الإدارة الإبداعية. } \\
& \text { V- قضايا في الحقوق المعلوماتية. } \\
& \text { 1- تصميم مواقع النشر الإلكترونية. }
\end{aligned}
$$

ومن أجل تغطية المؤسسـة التعليمية مجالات المعرفة يتحتم وضـع مجموعة من

$$
\text { المبادئ لعل من أهمها ما يلي : أهن }
$$

أ) إدارة المعرفة تتطلب الاستثمار في عدد من الأنشطة مثل (rr):

$$
\text { تصرير الملفات وتحميلها على قواعد معلومات. }
$$

تطوير بنية أساسية لمعالجة المعلومات التي تسهم في تطوير المعرفة. تعليم العاملين وتدريبهم على تبادل واستخدام المعلومات.

ب) الإدارة الفاعلة للمعرفة تتطلب تهجين العنصر البشري مع العنصر التقني في إنتاج المعرفة. ج) إدارة المعرفة لعبة سياسية تتطلب مفاوضات سرية ومداولات سياسية وحملات لولبية. د) إدارة المعرفة تحتاج إلى مدير من خلال إيجاد وظيفة لمدير المعرفة. هـ) إدارة المعرفة تتطلب خرائط للمعرفة وليس نماذج للمعرفة، كما تتطلب سوق للمعرفة أكثر من حاجتها إلى سلطة. و) عملية تبادل واستخدام المعارف تحتاج إلى تصرفات غير عادية نتيجة ما يرتبط بإدارة المعارف

$$
\text { من مخاطر وعقبات. }
$$

ز) إدارة المعرفة تحتاج إلى تحسين العمليات المعرفية وتطويرها سواء المتعلق منها بالإنسان أو بالآلة. ح) إدارة المعرفة عملية لا تعرف نقطة نهاية. ط) إدارة المعرفة تتطلب عقود تبادل المعرفة.

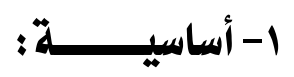

وإدارة المعرفة أساسية وليست موضـة، فالمعرفة هي إحدى أساسيات الموجودات غير الملموسة في عمليات المؤسسة، وتزداد منتجات وخدمات العديد من المؤسسات بوجود الابتكارات، لذا فإن إدارة المعرفة موجودة لتبقى. 


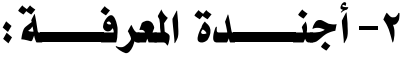

تتكون أجندة المعرفة من فرق المعرفة وقواعد المعرفة، مثل محركات البحث وأدوات الاستكثاف التي ساهم الذكاء الصناعي في توضيحها، والأدوات التصورية التي تخدم الأسواق المالية، وأدوات التعاون والمؤتمرات وغيرها، ومراكز المعرفة التي تقوم بمهام متعددة مثل تحديث

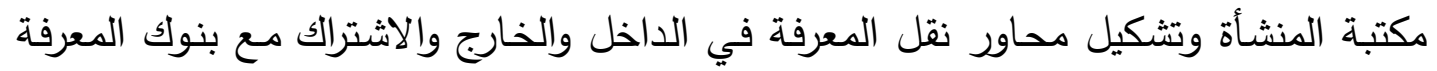
العالمية، والمؤسسة المتعلمة التي تقوم بمؤسسة ثقافية فيها التعلم التنظيمي والنمو من خلال إبداع وتطوير المؤسسة المتعلمة والتركيز على العامل الإنساني كعامل نجاح فيها تفوق أهميته الاهتمام بالتقنية، وجماعات الممارسة وهي مجموعة من الأفراد ممن يتثاركوا في الاهتمام ويواجهوا مشاكل

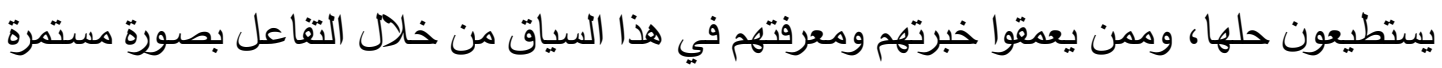

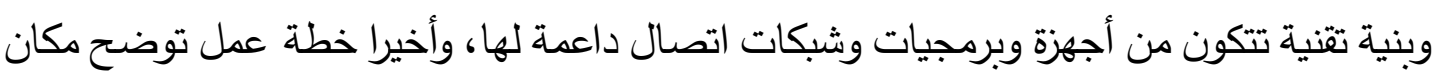
المؤسسة الآن والطريق الموصل لمستقبلها المنظور (rV)

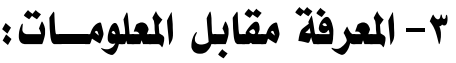

للتقريق بين المعرفة والمعلومات لا بد من البدء بحجر الأساس وهو البيانات، عبارة عن

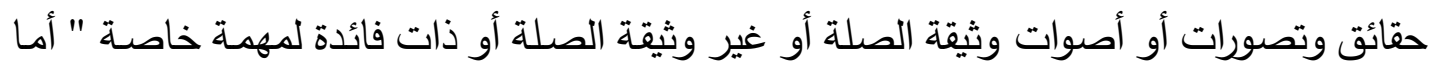
المعلومات فهي بيانات لها شكل ومحتوى يناسب استخدام خاص ويتم تحويل البيانات إلى

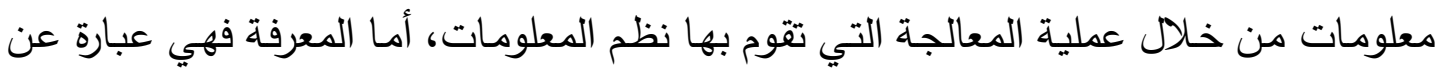
توليفة من المواهب والأفكار والقواعد والإجراءات التي تقود النشاطات والقرارات.

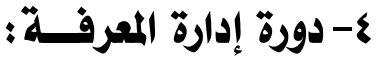

تبدأ مراحل دورة إدارة المعرفة بجمع المعرفة ثم تصنيفها ثم تتظيمها وتخزينها ثم المشاركة والنشر ثم الوصول والاستخدام ثم الإيجاد (التكوين) ثم التعريف وهكذا تستمر العملية.

\section{ه- قيـــــادة المعرفــــة}

توجد صفات متعدة لقادة المعرفة لابد من توفرها منها أن يملك هؤلاء القادة رؤية واضحة وموقف ذو قيمة حول مشاركة الممارسة الفضلى والابتكار الأسرع، وإعادة استخدام المعرفة،

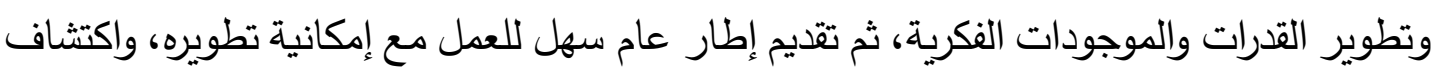
الرواد في العمل واستخدامهم كوكلاء تغيير ، وتسهيل شبكة الوصول إلى الخبراء من خلال تعزيز الاتصالات الممتازة الداخلية والخارجية(^^). 
تطبيق المعرفة هو الذي يحكم من خلاله على فعالية وفائدة المعرفة وخاصسة في العملية الإستراتيجية المتعلقة في تحقيق الجودة العالية للمنتجات والخدمات لمقابلة حاجات الزبائن، لذا

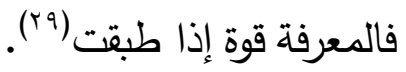

\section{خامساً: نمساذج إدارة المعسفهـة بالمؤسسة التعلبمية:}

توجد نماذج مختلفة لإدارة المعرفة حيث أن هذه النماذج اتخذت أشكالا عديدة وعروضـاً مبسطة للمعرفة، وكان الهدف من ذلك توجيه المؤسسة لبناء استراتيجيات معرفية تساعدها على لهـ تحقيق أهدافها وحل مشكلاتها وحتى تتماشى مع تغيرات العصر ، بالإضافة إلى الاستفادة من أكبر قدر من العاملين (رأس المال البشري للمؤسسة) حتى تصل هذه المؤسسة إلى مرحلة الإبداع والمنافسة، ومن هذه النماذج ما يلي :

أ) نموذج مالهوترا Molhotr

قدم مالهوترا نموذج يتكون من ثلاث مراحل وهي كالتالي (·r):

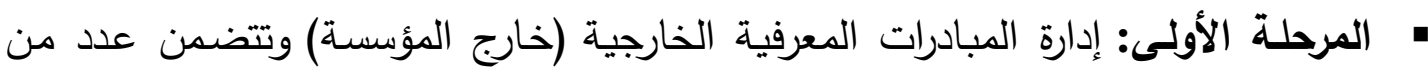

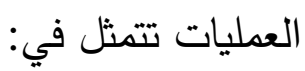
1 - اكتساب المعرفة من العملاء. r- تزويد العملاء بمعارف إضافية.

r- توليد موارد مالية جديدة من المعرفة المتوفرة. • المرحلة الثانية: إدارة المبادرات المعرفية الداخلية (داخل المؤسسة) وتتضمن عدد من

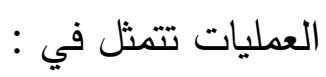
1 - بناء ثقافة لتبادل المعرفة. r- برعة توزيع وتخزين المعرفة التكتيكية. ب- قياس عمليات تطوير المعرفة. المرحلة الثالثة: إدارة الكفايات المعرفية، وتتضمن العمليات التالية: 1 - إيجاد مسارات وظيفية مبنية على المعرفة. Y- توفير بيئة مصغرة لنقل المعرفة. ب- دعم التعلم بتقنية المعلومات. ع - - التعلم من المواقف. 


\section{: Marquqrdt بموذج ماركوردت}

اقترح ماركوردت مدخل لإدارة المعرفة في المؤسسة ويتألف من ست مراحل تغطي عملية نقل المعرفة إلى المستخدم من خلال الخطوات التالية('آ):

$$
\text { צ - ا - الاكتساب. }
$$

\section{ج) نموذج ويج Wiig لإدارة المعرفة : ويندرج تحته ما يلي :}

1ا) نـموذج دورة تطور المعرفة المؤس1111.

يتكون نموذج دورة تطور المعرفة المؤسسية من خمس مراحل أساسية وهي (r؟r): 1- مرحلة تطوير وإعداد المعرفة : يجري تطوير المعرفة وإعدادها من خلال عمليات التعليم والإبداع والابتكار ، ومن خلال جهود البحث التي تهدف إلى جلب واستيراد المعرفة من

$$
\text { خارج المؤسسة. }
$$

ץ - مرحلة اكتساب المعرفة : في هذه المرحلة تتم السيطرة على المعرفة واكتسابها وتخزينها والاحتفاظ بها من أجل الاستخدام وعمليات المعالجة المختلفة من أجل تحقيق عمليات

$$
\text { الرفع من خلال المعرفة. }
$$

ب-مرحلة غربلة وتهذيب المعرفة : في هذه المرحلة يجري تتظيم المعرفة وتحويلها إلى أشكال مفيدة للمؤسسة، وهنا قد تحول المعرفة إلى مواد مكتوبة أو إلى قواعد معرفة وهذا يجعل المعرفة قادرة على تحقيق المنافع للمؤسسة. ع - مرحلة توزيع ونشر المعرفة : خلال هذه المرحلة يجري توزيع ونشر المعرفة حتى تصل إلى كل مركز وكل نقطة من نقاط العمل، وتجري عملية التوزيع والنشر عن طريق التعليم والبرامج التدريسية والنظم المسندة بالمعرفة وشبكات الأعمال الخبيرة، ويتم توزيع ونشر المعرفة لتشمل الأفراد والإجراءات والتقنيات والمنتجات (سلع وخدمات). ه- مرحلة الرفع المعرفي : في هذه المرحلة تكون المؤسسة قد تبنت وطبقت المعرفة كمحصلة للمراحل الأربعة السابقة، وهنا تبدأ المؤسسة تحقق ميزة الرفع المعرفي، وهذه الميزة تشبه ميزة الرفع المالي (الرافعة المالية في حقل الإدارة المالية)، وهي تحقق للمؤسسة عمليات تعلم أفضل وتزبد عمليات الابتكار والإبداع، وتكون أساسًا متينًا للميزة التنافسية. 


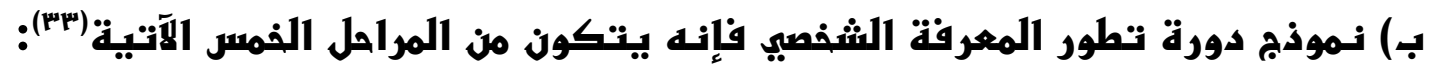
ا - مرحلة المعرفة الكامنة غير المدركة : هذه المعرفة تكون في الأغلب غير واضحة وغير

مفهومة بصورة جيدة، وهي عادة تعبر عن لمحة عابرة وانطباع أولي عن مفهوم جديد. r - مرحلة المعرفة المثالية : يكون جزء من هذه المعرفة معروفًا وواضحًا ويجري العمل معه بصورة واضحة، أمسا أغلب هذه المعرفة والتي تتعلق بالرؤى والنماذج العقلية فإنها ليست معروفة بصورة كافية وتكون ضمنية ويكون بلوغها والوصول إليها في صورة غير واضحة، ويمكن القول أن المقارنة المرجعية تقع ضمن هذه المرحلة. ب-مرحلة المعرفة النظامية : تتعلق هذه المعرفة بالنظم الأساسية واستراتيجيات حل المشكلات، وتتعلق بالمبادئ العامة، وبتعبير آخر فإن هذه المرحلة تتعلق بالمعرفة الصريحة الواضحة المعروفة جيدا، وهذه المعرفة يطلق عليها أحيانًا المعرفة العميقة ويجري استخدامها من أجل بناء النظم المسندة بالمعرفة. ع - مرحلة المعرفة الواقعية (العملية) : هذه المرحلة تتعلق بالمعرفة بصناعة القرارات وهي معرفة تكون في الأغلب معرفة صريحة، وتتعلق بدعم القرارات والأعمال اليومية، وتستخدم بصورة واضحة، وتقع عمليات التتريب ضمن هذه المرحلة.

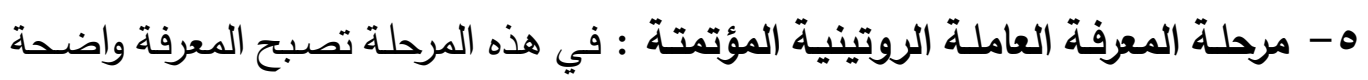
بصورة كافية إلى درجة تجعل من الممكن أتمتة هذه المعرفة، وتستخدم المعرفة في هذه المرحلة من أجل أداء المهام بصورة مؤتمتة وبصورة روتينية دون الحاجة إلى إعطاء مبررات وشرح الأسباب.

\section{سادساً: كيفية تدميق الاستفادة من نماذج إدارة المعرفهة للمؤسسهة التعليميسة في}

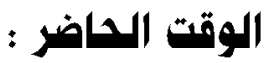

يمكـن للمؤسســـة التعليميــة أن تحقـق الاســففادة المنشــودة مــن نمــاذج إدارة المعرفة عن طريق (عَ): توليد المعرفة على اعتبار أنها تسهم في إثراء المؤسسة التعليمية وتساعد هذه المؤسسة على تحقيق الميزة التنافسية التي تتشدها مع المؤسسات المناظرة. 
تخزين المعرفة والتي بدورها تؤدي إلى تكوين ذاكرة تتظيمية للمؤسسة يتم من خلالها استثمار مواردها وتوظيف الإمكانات المتاحة لها واسترجاع المعلومات في الوقت الذي تريده المؤسسة التعليمية.

تطبيق المعرفة والتي تركز على استخدام المعرفة وتطبيقها في الأطر المتعارف عليها مـن قبـل المؤسسـة التعليميـة والتـي تسـهم بـدورها في تطوير الأنشطة المختلفـة بهـا وتساعدها أيضاً على الارتقاء بمكانتها بين المؤسسات المناظرة مع الأخذ في الاعتبار أن إدارة المعرفة تساعد المؤسسة التعليمية على: • (عديد مفتاح المعرفة التنظيمية. • تحديد مهمة المعرفة من خلال التحليل الوظيفي لكافة وظائف المؤسسة. • تحديد دور المعرفة في إنجاز أدوار العاملين. • تحديد مشاكل المعرفة وكيفية مواجهتها على ضوء الموارد المتاحة. • توظيف الــوارد البشرية المتاحسة على كل مجـالات العمل بالمؤسسـة مـع مراعـاة

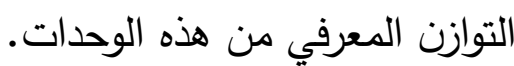




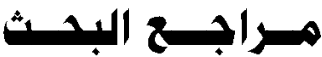

(1) عبد الفتاح المغربي: نظم إدارة المعلومـات الإداربـة، المكتبـة العصـرية للطباعـة والتوزيـع،

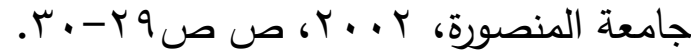

(Y) صـلاح الـدين الكبيسـي، خضـير خـام: إدارة المعرفـة، المنظمـة العربيـة لــلإدارة، القــاهرة،

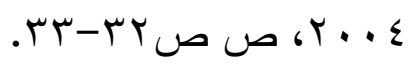

(r) ليث عبد الله القهيوي: إستراتيجية إدارة المعرفة، دار الحامد للنشر والتوزيع، طا، عمان،

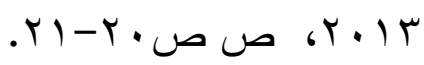

(ع) عمر احمد هشري: إدارة المعرفة الطريق إلى التمييز والربـادة، دار الصفاء للنشر والتوزيع،

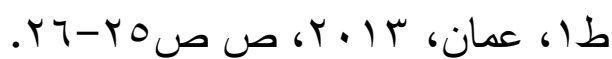

(0) محمد عواد الزيادات: اتجاهات معاصرة في إدارة المعرفة، دار الصفاء للنشر والتوزيع، طا،

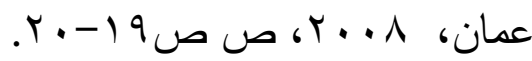

محمد عشماوي: الإدارة الإستراتيجية في تتمية موارد بشربة، منشاة المعارف الإسكندرية،

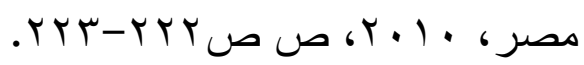

(V)

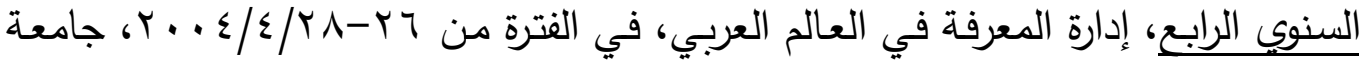

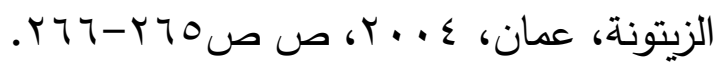

(^) سـلامة عبـد العظيم حسين، حسـن البيلاوي: إدارة المعرفـة في التعليم، دار الوفـاء لـنيا

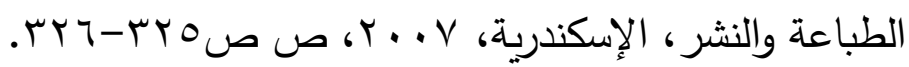

(9) أميرة هاتف حداوي الجنابي، اثر إدارة المعرفة الزبون في تحقيق التفوق التنافسي، رسالة ماجستير غير منشورة، تخصص علوم إدارة الأعمال، كلية الإدارة والاقتصاد، جامعة الكوفة،

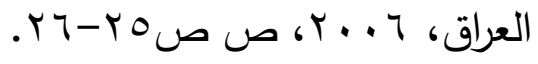


( • (1) إيمان قحموش، دور إدارة المعرفة في تحسين تسيير علاقات الزبائن، رسالة ماجستير غير منشورة، تخصص إدارة المعرفة والمعارف، كلية العلوم الاقتصادية والتجاريـة، جامعة محمد

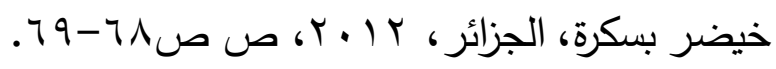

(11) Maryam Alavi: Knowledge Management And Knowledge Systems,

Knowing Medford, New Jersey, 2007, PP. 121-122.

(Y Y) أكرم سالم الجنابي: إدارة المعرفة في بناء الكفايات الجوهربة، مؤسسة الوراق للنشر

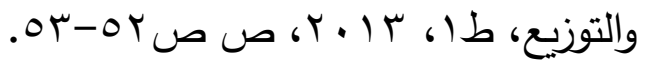

(r (1) ربحي مصطفى عليان: إدارة المعرفة، دار الصفاء للنشر والتوزيع، طا، عمان، A....؟،

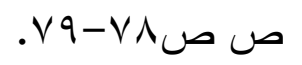

( ) ( ) باير حبيب، بلجوم فريد: دور إدارة المعرفة في تتمية الموارد البشرية، ملتقى دولي حول رأس

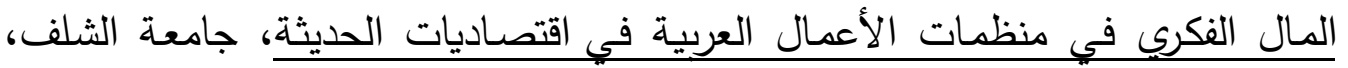

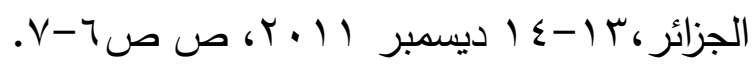

(10) عـلاء فرحان، أميرة الجنابي: إدارة المعرفة، دار الصفاء للنشر والتوزيع، طا،

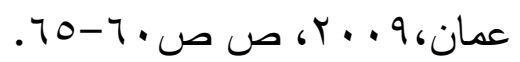

(16) Maryam Alavi \& E. Leidner Dorothy: Knowledge Management :Systems: Issues, Challenges, and Benefits (in) Knowledge Managmenet Systems Theory and Practice, Edited by sturat Bares. Thomson Learning, 2012, PP. 22-23.

(IV) رباجزا جميل المحاميد: دور إدارة المعرفة في تحقيق جودة التعليم العالي، رسالة ماجستير غير منشورة، تخصص إدارة أعمال، كلية علوم إدارية والمالية، جامعة الشرق الأوسط

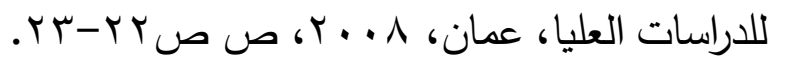

(1) (1) ياسر بن عبد الله بن تركي العتيبي: إدارة المعرفة وإمكانية تطبيقها في الجامعة السعودية،

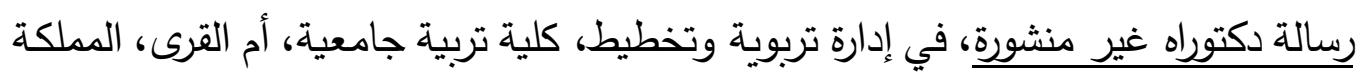

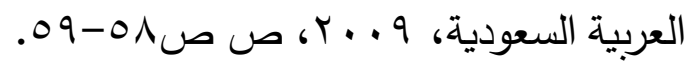


(9 (1) طارق فيصل التميمي: أساسيات إدارة المعرفة، رسالة ماجستير غير منشورة، الأكاديمية

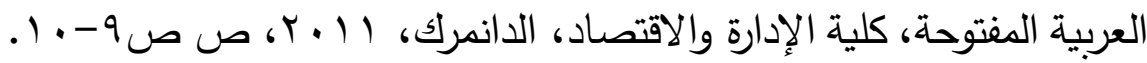

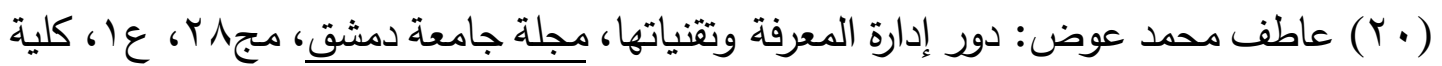

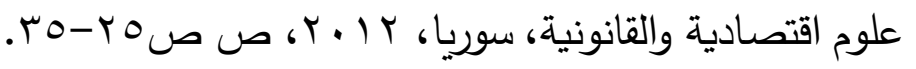

(21) Verna Allen: The Knowledge Evolution, Butterworth-Heinemann: MA, Boston, 2013, PP. 78-79.

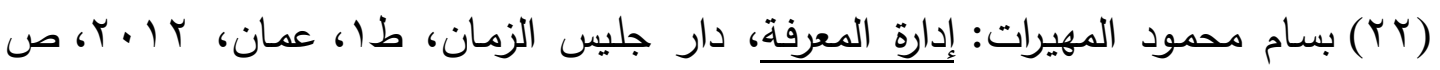

$$
.0 \cdot-\leqslant 90
$$

(YT) خضر مصباح إسماعيل طيطي: إدارة المعرفة التحديات والتقيات والحلول، دار الحامد،

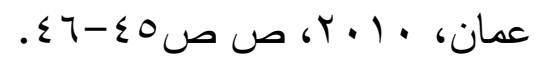

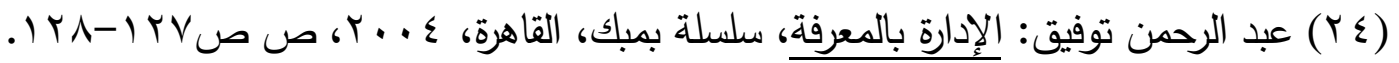

(Y0) جويبر مـاطر الثبيتي: إدارة المعرفـة والاستثمار في رأس المـال المعرفي، مجلـة التربيـة

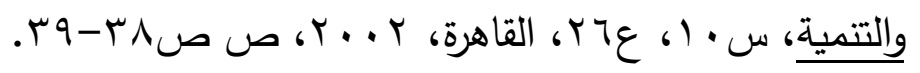

(T (Y) كيـث دفلين: الإنسـان والمعرفـة في عصـر المعلومـات، ترجمـة شـادن اليـافي، مكتبـة

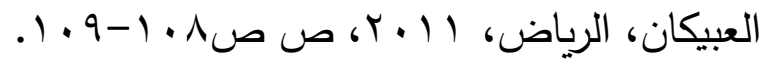

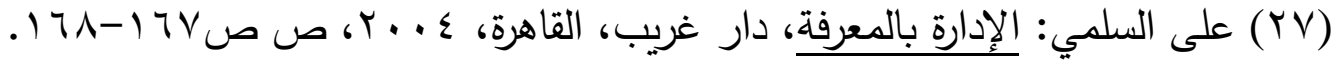

( أحمد كامل الرشيدي: مشكلات الإدارة المدرسية في الألفية الثالثة - رؤبـة تربوبـة جديدة،

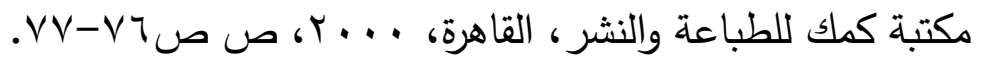

(Y9) رشاد الساعد، حسين حريخ: إدارة المعرفة وتكنولوجيا المعلومات، دار اليازوري للطباعة

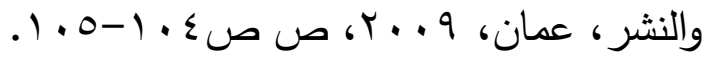

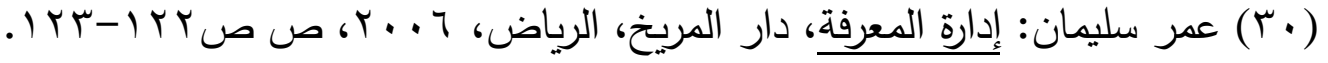


(T) حسن شحاته: مداخل إلى تعليم المستقبل في الوطن العربي، تقديم حامد عمار، الدار

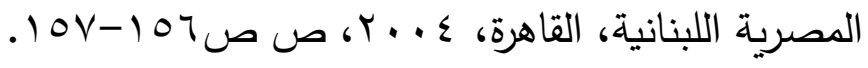

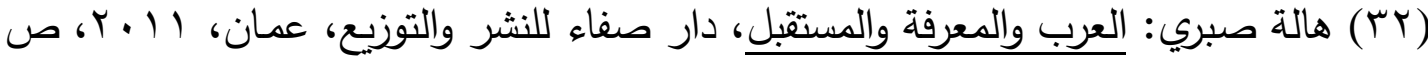

$$
\text { صLTIVTR }
$$

(Tr) ياسر عبد الله: إدارة المعرفة وإمكانية تطبيقها في الجامعات السعودية - دراسة تطبيقية

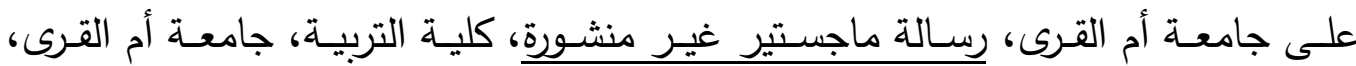

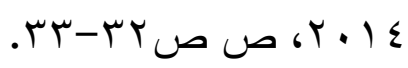

(؟) ساهرة الملاكك، أحمد الأثري: إدارة المعرفة ودورها في دعم المهارات التتموية للمنظمات،

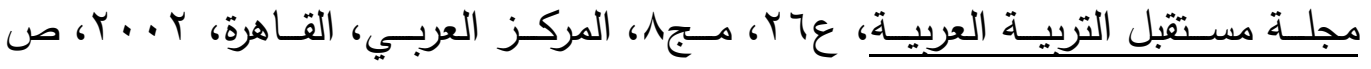

. $1 \leqslant V-1 \leqslant 7$ 